\title{
USE OF OXALIC ACID FOR SCREENING INTACT SUNFLOWER PLANTS FOR RESISTANCE TO Sclerotinia IN VITRO
}

\author{
Dragana Vasić*, Dragan Škorić, Ksenija Taški, Ljiljana Stošić \\ Institute of Field and Vegetable Crops, Maksima Gorkog 30, \\ 21000 Novi Sad, Yugoslavia
}

Received: November 08, 2001 Accepted: June 03, 2002

\author{
SUMMARY
}

Three sunflower inbred lines differing in resistance to Sclerotinia were grown on nutritive medium supplemented with different concentrations of oxalic acid. After two weeks of culture, plant height, fresh and dry weight of above-ground part, length of first pair of leaves, root length, and fresh and dry weight of root were measured. Data obtained were analysed using ANOVA and LSD test. Out of the tested parameters, plant height and root length were found to be the most reliable indicators of plant resistance/susceptibility to Sclerotinia. Based on the data obtained, oxalic acid concentrations of $4 \mathrm{mM}, 3 \mathrm{mM}$ and $2 \mathrm{mM}$ were chosen for further work in which test would be done on a larger number of genotypes.

Key words: sunflower, Sclerotinia resistance, in vitro screening, oxalic acid

\section{INTRODUCTION}

The white rot disease caused by the fungus Sclerotinia sclerotiorum is the major disease of sunflower in countries with the humid climate, while in countries with the moderate climate, it causes yield loss in rainy years (Škorić and Rajčan, 1992). It is a facultative polyphagous parasite that attacks a variety of wild plants and agricultural crops. There are no suitable cultural control methods against it (Lumsden, 1979) and no resistant genotypes of cultivated sunflower have yet been found or developed. Breeding work is complicated since there are several forms of disease and the mechanisms of tolerance to the various forms are controlled by different genetic and other processes (Roberts et al., 1987).

De Bary was the first who associated oxalic acid with Sclerotinia infection (Lumsden, 1979). Later, Noyes and Hankock (1981) demonstrated its importance as a factor in the pathogenicity of this fungus, while Hartman et al. (1988) found

* Corresponding author: e-mail: vasicd@ifvcns.ns.ac.yu 
correlation between oxalic acid production and virulence of different isolates of Sclerotinia.

For an early selection for the disease resistance, a laboratory method is highly desirable. The larger number of individuals that can be screened and the limited space that is needed make the application of in vitro selection an attractive approach. There have been several attempts to create a bioassay in which resistance to oxalic acid would be used as an indicator of resistance to Sclerotinia (Hartman et al., 1988; Noyes and Hancock, 1981; Raducanu and Soare, 1992; Tu, 1985; Vasić et al., 1999). Whole plants or their parts were used, and correlation was found between field susceptibility/resistance of genotypes tested to Sclerotinia and reaction of the explants of the same genotypes when grown on a medium to which oxalic acid was added.

In this paper we describe a method for in vitro screening for resistance to Sclerotinia by culture of intact sunflower plants in the presence of oxalic acid.

\section{MATERIAL AND METHODS}

Inbred lines PR-ST-3B, tolerant to Sclerotinia on the head, $\mathrm{CMS}_{1}$-90A, susceptible to Sclerotinia on the root, and OD-3369A, susceptible to Sclerotinia on the stem, were used in the experiment (Škorić, personal communication). Institute of Field and Vegetable Crops, Novi Sad, Yugoslavia, provided seeds of all lines.

Seeds were surface sterilized by soaking in $5 \%$ solution of commercial bleach for 20 minutes, washed with distilled water and dehulled. Dehulled seeds were sterilized again by dipping in $70 \%$ ethanol for one minute followed by soaking in commercial bleach for ten minutes. They were then rinsed three times with sterile distilled water and placed in $250 \mathrm{ml}$ Erlenmeyer flasks with $80 \mathrm{ml}$ of MS medium (Murashige and Skoog, 1962) supplemented with $5 \mathrm{~g} \mathrm{l}^{-1}$ of sucrose and $6 \mathrm{~g} \mathrm{l}^{-1}$ of agar, pH 5.7, and different concentrations of oxalic acid (Tables 1, 2 and 3). Seeds were further cultured in the light of intensity of $34 \mu \mathrm{E} \mathrm{m} \mathrm{m}^{-1}$, photoperiod 16:8 (light:dark) and temperature $25^{\circ} \mathrm{C}$.

After two weeks of culture following parameters were measured: plant height, fresh and dry weight of above-ground part, length of first pair of leaves, root length, fresh and dry weight of root. The experiment was set in four repetitions, and three cycles. In each cycle different set of oxalic acid concentrations was tested. For statistical analysis, the data obtained for each parameter were expressed as percentage of the respective mean of control (100\%). Data were analyzed using ANOVA and LSD test. 


\section{RESULTS AND DISCUSSION}

Primary choice of oxalic acid concentrations was made based on the research of Vasić et al. (1999) done on sunflower protoplasts (Table 1). In the work of Vasić et al. (1999), the concentration of $1 \mathrm{mM}$ of oxalic acid discriminated well between the resistant and susceptible genotypes. This was not the case in our study, where concentrations between 1.2 and $1.8 \mathrm{mM}$ did not have negative effect on the tested parameters of either resistant or susceptible genotypes. In some cases, even stimulating effect on some parameters was observed (Table 1). Higher selectivity of given concentration of oxalic acid in the case of protoplasts can be explained by their greater sensitivity to osmotic shocks, which oxalic acid as a very strong chelator of calcium and other cations (Lumsden, 1979) provokes. Stimulating effect of small concentrations of stress agents in in vitro culture was observed also in the experiments with herbicides (Olofsdotter et al., 1994), and is thought to be a consequence of phenomenon that stress agents when present in small concentrations act as nutrients (Vasić et al., 1997).

Table 1: Reaction of tested genotypes on treatment with different concentrations of oxalic acid. Within the column and the genotype means followed by different letter differ. Legend for traits: $\mathrm{h}$ - plant height, fm - fresh weight of above-ground part, dm - dry weight of above-ground part, 1 - length of first pair of leaves, $\mathrm{rm}$ - fresh weight of root, rdm dry weight of root, rl - root length.

\begin{tabular}{clccccccc}
\hline \multirow{2}{*}{ Genotype } & \multirow{2}{*}{ Conc $\mathrm{mM}$} & \multicolumn{7}{c}{ Trait $(\%)$} \\
\cline { 3 - 9 } & & $\mathrm{h}$ & $\mathrm{fm}$ & $\mathrm{dm}$ & $\mathrm{l}$ & $\mathrm{rfm}$ & $\mathrm{rdm}$ & $\mathrm{rl}$ \\
\hline PR-ST-3B & Control & $100.0 \mathrm{a}$ & $100.0 \mathrm{a}$ & $100.0 \mathrm{a}$ & $100.0 \mathrm{a}$ & $100.0 \mathrm{a}$ & $100.0 \mathrm{a}$ & $100.0 \mathrm{a}$ \\
& 1.2 & $79.7 \mathrm{a}$ & $97.6 \mathrm{a}$ & $108.8 \mathrm{a}$ & $105.8 \mathrm{a}$ & $87.6 \mathrm{a}$ & $98.7 \mathrm{a}$ & $94.5 \mathrm{a}$ \\
& 1.4 & $78.2 \mathrm{a}$ & $102.9 \mathrm{a}$ & $105.6 \mathrm{a}$ & $120.6 \mathrm{a}$ & $77.0 \mathrm{a}$ & $74.9 \mathrm{a}$ & $103.8 \mathrm{a}$ \\
& 1.6 & $90.7 \mathrm{a}$ & $101.1 \mathrm{a}$ & $95.8 \mathrm{a}$ & $138.9 \mathrm{a}$ & $81.4 \mathrm{a}$ & $85.6 \mathrm{a}$ & $119.8 \mathrm{a}$ \\
& 1.8 & $77.4 \mathrm{a}$ & $98.9 \mathrm{a}$ & $101.4 \mathrm{a}$ & $92.9 \mathrm{a}$ & $72.9 \mathrm{a}$ & $69.8 \mathrm{a}$ & $94.4 \mathrm{a}$ \\
& LSD $_{0.01}$ & 42.6 & 22.0 & 22.9 & 52.2 & 33.0 & 38.2 & 29.9 \\
\hline $\mathrm{CMS}_{1}$-90A & control & $100.0 \mathrm{a}$ & $100.0 \mathrm{bc}$ & $100.0 \mathrm{a}$ & $100.0 \mathrm{a}$ & $100.0 \mathrm{~b}$ & $100.0 \mathrm{bc}$ & $100.0 \mathrm{a}$ \\
& 1.2 & $102.1 \mathrm{a}$ & $127.9 \mathrm{a}$ & $91.8 \mathrm{a}$ & $113.9 \mathrm{a}$ & $149.0 \mathrm{a}$ & $150.9 \mathrm{a}$ & $94.8 \mathrm{a}$ \\
& 1.4 & $95.9 \mathrm{a}$ & $107.0 \mathrm{~b}$ & $104.6 \mathrm{a}$ & $105.1 \mathrm{a}$ & $120.4 \mathrm{ab}$ & $105.4 \mathrm{bc}$ & $90.3 \mathrm{a}$ \\
& 1.6 & $92.9 \mathrm{a}$ & $86.5 \mathrm{c}$ & $81.9 \mathrm{a}$ & $94.4 \mathrm{a}$ & $97.1 \mathrm{~b}$ & $93.1 \mathrm{c}$ & $85.4 \mathrm{a}$ \\
& 1.8 & $92.8 \mathrm{a}$ & $118.5 \mathrm{ab}$ & $108.9 \mathrm{a}$ & $110.1 \mathrm{a}$ & $146.4 \mathrm{a}$ & $138.2 \mathrm{ab}$ & $92.4 \mathrm{a}$ \\
& $\mathrm{LSD}_{0.01}$ & 29.1 & 28.4 & 41.9 & 33.6 & 39.6 & 40.0 & 19.7 \\
\hline OD-3369A $^{2}$ & Control & $100.0 \mathrm{a}$ & $100.0 \mathrm{a}$ & $100.0 \mathrm{a}$ & $100.0 \mathrm{a}$ & $100.0 \mathrm{a}$ & $100.0 \mathrm{ab}$ & $100.0 \mathrm{a}$ \\
& 1.2 & $70.5 \mathrm{a}$ & $70.8 \mathrm{ab}$ & $80.4 \mathrm{ab}$ & $65.2 \mathrm{~b}$ & $68.5 \mathrm{a}$ & $53.9 \mathrm{~b}$ & $53.5 \mathrm{a}$ \\
& 1.4 & $64.8 \mathrm{a}$ & $63.2 \mathrm{~b}$ & $74.3 \mathrm{~b}$ & $53.9 \mathrm{~b}$ & $63.5 \mathrm{a}$ & $67.2 \mathrm{~b}$ & $81.7 \mathrm{a}$ \\
& 1.6 & $75.8 \mathrm{a}$ & $75.4 \mathrm{ab}$ & $76.9 \mathrm{ab}$ & $71.6 \mathrm{ab}$ & $85.6 \mathrm{a}$ & $73.8 \mathrm{~b}$ & 68.3 \\
& 1.8 & $99.4 \mathrm{a}$ & $73.9 \mathrm{ab}$ & $73.4 \mathrm{~b}$ & $74.2 \mathrm{ab}$ & $129.2 \mathrm{a}$ & $142.1 \mathrm{a}$ & $73.2 \mathrm{a}$ \\
& $\mathrm{LSD}_{0.01}$ & 47.9 & 30.4 & 24.5 & 29.9 & 78.1 & 49.3 & 58.4 \\
\hline
\end{tabular}

In the second cycle of the experiment, a set of oxalic acid concentrations from 2 to $4 \mathrm{mM}$ was tested (Table 2). The reaction of the tested genotypes to these concen- 
trations was more similar to the reaction in the field than in the previous set. This especially stands for concentrations of 2,3 and $4 \mathrm{mM}$. The resistant genotype did not react to the treatment with the $2 \mathrm{mM}$ of oxalic acid, while this concentration had negative effect on height of both susceptible genotypes (Table 2). The highest concentration $(4 \mathrm{mM})$ had negative effect on all tested parameters of the susceptible genotypes, and height, leaf length and dry and fresh weight of root of the resistant genotype (Table 2). Plant height could be considered as a potential parameter of general resistance/susceptibility to Sclerotinia as it was affected it in both susceptible genotypes in a similar manner. Another interesting parameter is root length. Beside small coefficient of variability (data not shown), it was not affected by treatment in the resistant genotype and was significantly decreased in the susceptible ones in the presence of the highest concentration of oxalic acid. Concentration of $3 \mathrm{mM}$ was interesting as it probably could be used to discriminate resistance/susceptibility to different forms of Sclerotinia, since the reaction to treatment in the two susceptible genotypes differed the most when this concentration was used (Table 2).

Table 2: Reaction of tested genotypes on treatment with different concentrations of oxalic acid. Within the column and the genotype means followed by different letter differ. Legend for traits: $\mathrm{h}$ - plant height, fm - fresh weight of above-ground part, dm - dry weight of above-ground part, 1 - length of first pair of leaves, rfm - fresh weight of root, rdm dry weight of root, $\mathrm{rl}$ - root length.

\begin{tabular}{llccccccc}
\hline Genotype & Conc. $\mathrm{mM}$ & \multicolumn{7}{c}{ Trait (\%) } \\
\cline { 3 - 8 } & & $\mathrm{h}$ & $\mathrm{fm}$ & $\mathrm{dm}$ & $\mathrm{l}$ & $\mathrm{rfm}$ & $\mathrm{rdm}$ & $\mathrm{rl}$ \\
\hline PR-ST-3B & Control & $100.0 \mathrm{a}$ & $100.0 \mathrm{a}$ & $100.0 \mathrm{a}$ & $100.0 \mathrm{a}$ & $100.0 \mathrm{a}$ & $100.0 \mathrm{a}$ & $100.0 \mathrm{a}$ \\
& 2.0 & $75.3 \mathrm{ab}$ & $97.7 \mathrm{a}$ & $116.9 \mathrm{a}$ & $96.6 \mathrm{ab}$ & $75.9 \mathrm{ab}$ & $82.0 \mathrm{ab}$ & $128.9 \mathrm{~s}$ \\
& 2.5 & $63.0 \mathrm{bc}$ & $112.9 \mathrm{a}$ & $137.5 \mathrm{a}$ & $116.5 \mathrm{a}$ & $68.4 \mathrm{ab}$ & $81.0 \mathrm{ab}$ & $124.2 \mathrm{a}$ \\
& 3.0 & $58.2 \mathrm{bc}$ & $98.7 \mathrm{a}$ & $106.1 \mathrm{a}$ & $57.9 \mathrm{bc}$ & $77.9 \mathrm{ab}$ & $82.3 \mathrm{ab}$ & $119.4 \mathrm{a}$ \\
& 3.5 & $43.8 \mathrm{c}$ & $87.4 \mathrm{a}$ & $93.8 \mathrm{a}$ & $11.9 \mathrm{~d}$ & $48.9 \mathrm{~b}$ & $54.5 \mathrm{~b}$ & $105.7 \mathrm{a}$ \\
& 4.0 & $52.5 \mathrm{c}$ & 96.9 & $102.7 \mathrm{a}$ & $18.7 \mathrm{~cd}$ & $59.9 \mathrm{~b}$ & $68.8 \mathrm{~b}$ & $103.8 \mathrm{a}$ \\
& LSD $_{0.01}$ & 24.7 & 32.3 & 45.3 & 41.1 & 37.5 & 29.0 & 38.34 \\
\hline CMS $_{1}$-90A & Control & $100.0 \mathrm{a}$ & $100.0 \mathrm{a}$ & $100.0 \mathrm{ab}$ & $100.0 \mathrm{a}$ & $100.0 \mathrm{a}$ & $100.0 \mathrm{a}$ & $100.0 \mathrm{a}$ \\
& 2.0 & $81.9 \mathrm{~b}$ & $40.3 \mathrm{~b}$ & $92.2 \mathrm{~b}$ & $88.7 \mathrm{a}$ & $86.9 \mathrm{ab}$ & $93.8 \mathrm{ab}$ & $91.5 \mathrm{a}$ \\
& 2.5 & $81.6 \mathrm{~b}$ & $44.3 \mathrm{~b}$ & $102.4 \mathrm{ab}$ & $91.7 \mathrm{a}$ & $86.4 \mathrm{ab}$ & $87.2 \mathrm{ab}$ & $95.5 \mathrm{a}$ \\
& 3.0 & $54.5 \mathrm{c}$ & $35.3 \mathrm{~b}$ & $88.6 \mathrm{~b}$ & $53.1 \mathrm{~b}$ & $67.7 \mathrm{~b}$ & $85.2 \mathrm{ab}$ & $82.2 \mathrm{ab}$ \\
& 3.5 & $50.9 \mathrm{~cd}$ & $50.1 \mathrm{~b}$ & $88.1 \mathrm{~b}$ & $28.8 \mathrm{c}$ & $61.5 \mathrm{~b}$ & $72.8 \mathrm{~b}$ & $70.7 \mathrm{~b}$ \\
& 4.0 & $37.1 \mathrm{~d}$ & $44.6 \mathrm{~b}$ & $115.2 \mathrm{a}$ & $5.9 \mathrm{c}$ & $67.8 \mathrm{~b}$ & $93.8 \mathrm{ab}$ & $69.5 \mathrm{~b}$ \\
& LSD $_{0.01}$ & 14.2 & 33.5 & 21.7 & 23.35 & 27.2 & 24.9 & 20.8 \\
\hline OD-3369A & Control & $100.0 \mathrm{a}$ & $100.0 \mathrm{a}$ & $100.0 \mathrm{a}$ & $100.0 \mathrm{a}$ & $100.0 \mathrm{a}$ & $100 / 0 \mathrm{a}$ & $100.0 \mathrm{a}$ \\
& 2.0 & $57.2 \mathrm{~b}$ & $70.2 \mathrm{ab}$ & $83.8 \mathrm{a}$ & $63.6 \mathrm{bc}$ & $79.1 \mathrm{ab}$ & $65.4 \mathrm{ab}$ & $65.4 \mathrm{~b}$ \\
& 2.5 & $71.1 \mathrm{~b}$ & $87.9 \mathrm{ab}$ & $101.6 \mathrm{a}$ & $73.1 \mathrm{~b}$ & $86.5 \mathrm{ab}$ & $65.4 \mathrm{ab}$ & $74.0 \mathrm{ab}$ \\
& 3.0 & $58.8 \mathrm{~b}$ & $72.1 \mathrm{ab}$ & $79.1 \mathrm{a}$ & $45.6 \mathrm{~cd}$ & $75.6 \mathrm{ab}$ & $61.6 \mathrm{ab}$ & $62.1 \mathrm{~b}$ \\
& 3.5 & $61.9 \mathrm{~b}$ & $85.5 \mathrm{ab}$ & $103.2 \mathrm{a}$ & $54.1 \mathrm{bcd}$ & $85.4 \mathrm{ab}$ & $72.7 \mathrm{ab}$ & $63.2 \mathrm{~b}$ \\
& 4.0 & $51.4 \mathrm{~b}$ & $66.6 \mathrm{~b}$ & $90.2 \mathrm{a}$ & $31.4 \mathrm{~d}$ & $40.1 \mathrm{~b}$ & $41.5 \mathrm{~b}$ & $33.6 \mathrm{c}$ \\
& LSD $_{0.01}$ & 25.4 & 32.2 & 31.8 & 24.8 & 50.82 & 51.3 & 27.8 \\
\hline
\end{tabular}


Concentrations of oxalic acid higher than $4 \mathrm{mM}$ were found not to be reliable for the selection for Sclerotinia resistance as the results obtained were not in correlation with the field results (Table 3). All tested concentrations affected both resistant and susceptible genotypes. This is in disagreement with the results of Mouly (1989) who found concentrations of oxalic acid lower than $4.44 \mathrm{mM}$ not selective in bioassay with sunflower leaves. The same author recommended a concentration of $8.88 \mathrm{mM}$ as optimal.

Table 3: Reaction of tested genotypes on treatment with different concentrations of oxalic acid. Within the column and the genotype means followed by different letter differ. Legend for traits: $\mathrm{h}$ - plant height, fm - fresh weight of above-ground part, dm - dry weight of above-ground part, 1 - length of first pair of leaves, rfm - fresh weight of root, rdm dry weight of root, $\mathrm{rl}$ - root length.

\begin{tabular}{llccccccc}
\hline \multirow{2}{*}{ Genotype } & \multirow{2}{*}{ Conc. $\mathrm{mM}$} & \multicolumn{7}{c}{ Trait (\%) } \\
\cline { 3 - 9 } & & $\mathrm{h}$ & $\mathrm{fm}$ & $\mathrm{dm}$ & $\mathrm{l}$ & $\mathrm{rfm}$ & $\mathrm{rdm}$ & $\mathrm{rl}$ \\
\hline PR-ST-3B & Control & $100.0 \mathrm{a}$ & $100.0 \mathrm{~b}$ & $100.0 \mathrm{~b}$ & $100.0 \mathrm{a}$ & $100.0 \mathrm{a}$ & $100.0 \mathrm{a}$ & $100.0 \mathrm{a}$ \\
& 4.2 & $35.9 \mathrm{~b}$ & $115.6 \mathrm{a}$ & $129.3 \mathrm{a}$ & $56.1 \mathrm{~b}$ & $20.1 \mathrm{c}$ & $29.4 \mathrm{bc}$ & $58.2 \mathrm{bc}$ \\
& 4.4 & $27.9 \mathrm{bc}$ & $67.5 \mathrm{~d}$ & $92.5 \mathrm{c}$ & $50.8 \mathrm{bc}$ & $27.6 \mathrm{c}$ & $50.5 \mathrm{~b}$ & $45.0 \mathrm{~cd}$ \\
& 4.6 & $19.1 \mathrm{c}$ & $81.3 \mathrm{c}$ & $77.3 \mathrm{e}$ & $41.2 \mathrm{c}$ & $44.4 \mathrm{~b}$ & $15.6 \mathrm{c}$ & $82.2 \mathrm{ab}$ \\
& 4.8 & $20.2 \mathrm{c}$ & $45.5 \mathrm{e}$ & $85.7 \mathrm{~d}$ & $55.0 \mathrm{~b}$ & $14.9 \mathrm{~d}$ & $36.8 \mathrm{bc}$ & $17.8 \mathrm{~d}$ \\
& $\mathrm{LSD}_{0.01}$ & 10.8 & 5.2 & 4.2 & 12.0 & 15.5 & 22.7 & 34.5 \\
\hline $\mathrm{CMS}_{1}$-90A A & Control & $100.0 \mathrm{a}$ & $100.0 \mathrm{a}$ & $100.0 \mathrm{a}$ & $100.0 \mathrm{a}$ & $100.0 \mathrm{a}$ & $100.0 \mathrm{a}$ & $100.0 \mathrm{a}$ \\
& 4.2 & $26.1 \mathrm{~b}$ & $58.1 \mathrm{~b}$ & $74.8 \mathrm{~b}$ & $26.3 \mathrm{~b}$ & $26.3 \mathrm{~b}$ & $30.3 \mathrm{a}$ & $53.8 \mathrm{~b}$ \\
& 4.4 & $13.6 \mathrm{c}$ & $46.7 \mathrm{bc}$ & $63.8 \mathrm{bc}$ & $7.5 \mathrm{bc}$ & $12.1 \mathrm{~b}$ & $55.9 \mathrm{a}$ & $17.4 \mathrm{~b}$ \\
& 4.6 & $22.0 \mathrm{bc}$ & $42.8 \mathrm{c}$ & $61.0 \mathrm{c}$ & $4.5 \mathrm{c}$ & $30.7 \mathrm{~b}$ & $70.8 \mathrm{a}$ & $39.9 \mathrm{~b}$ \\
& 4.8 & $19.0 \mathrm{bc}$ & $56.6 \mathrm{bc}$ & $75.2 \mathrm{~b}$ & $14.6 \mathrm{bc}$ & $15.3 \mathrm{~b}$ & $60.7 \mathrm{a}$ & $21.1 \mathrm{~b}$ \\
& $\mathrm{LSD}_{0.01}$ & 9.2 & 14.6 & 11.9 & 2.9 & 26.6 & 106.1 & 43.2 \\
\hline OD-3369A A $^{2}$ & Control & $100.0 \mathrm{a}$ & $100.0 \mathrm{a}$ & $100.0 \mathrm{a}$ & $100.0 \mathrm{a}$ & 100.0 & $100.0 \mathrm{~b}$ & $100.0 \mathrm{a}$ \\
& 4.2 & $59.7 \mathrm{~b}$ & $80.2 \mathrm{~b}$ & $91.4 \mathrm{a}$ & $77.0 \mathrm{ab}$ & $61.9 \mathrm{c}$ & $93.8 \mathrm{~b}$ & $48.4 \mathrm{c}$ \\
& 4.4 & $39.2 \mathrm{~b}$ & $61.4 \mathrm{c}$ & $70.8 \mathrm{bc}$ & $61.3 \mathrm{~b}$ & $49.8 \mathrm{~d}$ & $72.6 \mathrm{c}$ & $53.7 \mathrm{c}$ \\
& 4.6 & $56.4 \mathrm{~b}$ & $69.1 \mathrm{bc}$ & $75.4 \mathrm{~b}$ & $55.9 \mathrm{bc}$ & $89.4 \mathrm{~b}$ & $126.0 \mathrm{a}$ & $77.1 \mathrm{~b}$ \\
& 4.8 & 27.1 & $42.9 \mathrm{~d}$ & $59.6 \mathrm{c}$ & $27.0 \mathrm{c}$ & $12.9 \mathrm{e}$ & $26.0 \mathrm{~d}$ & $23.4 \mathrm{~d}$ \\
& $\mathrm{LSD}_{0.01}$ & 39.6 & 18.4 & 13.2 & 33.1 & 9.3 & 12.6 & 12.7 \\
\hline
\end{tabular}

\section{CONCLUSION}

The results obtained in our study were in accordance with the results of Hartman et al. (1988), Raducanu and Soare (1992), and Vasić et al. (1999) who found correlation between the tolerance to oxalic acid in the calli and protoplasts and the resistance of field grown plants. Of the tested parameters, plant height and root length were found to be the most reliable indicators of plant resistance/susceptibility to Sclerotinia. Based on the data obtained, oxalic acid concentrations of $4 \mathrm{mM}$, $3 \mathrm{mM}$ and $2 \mathrm{mM}$ were chosen for further work in which test would be done on a larger number of genotypes. 


\title{
REFERENCES
}

Hartman, C.L., Donald, P.A., Secor, G.A. and Miller, J.F., 1988. Sunflower tissue culture and use in selection for resistance to Phoma macdonaldii and white mold (Sclerotinia sclerotiorum). Proceedings of the $12^{\text {th }}$ International Sunflower Conference, Novi Sad, Yugoslavia, pp. 347-351.

Lumsden, R.D., 1979. Histology and physiology of pathogenesis in plant diseases caused by Sclerotinia species. Phytopathology, 8: 890-895.

Mouly, A., 1989. Recherche de marqueur moleculares de la tolerance du tournesol a Sclerotinia sclerotiorum. Doctoral Thesis. UPS Toulouse, pp. 70.

Murashige, T. and Skoog, F., 1962. A revised medium for growth and bioassays with tobacco tissue cultures. Physiologia Plantarum, 15: 473-497.

Noyes, R.D. and Hancock, J.G., 1981. Role of oxalic acid in the Sclerotinia wilt of sunflower. Physiological Plant Pathology, 18: 123-132.

Olofsdotter, M., Olesen, A., Andersen, S.B. and Streibig, J.C., 1994. A comparison of herbicide bioassays in cell cultures and whole plants. Weed Res., 34: 387-394.

Raducanu, F. and Soare, G., 1992. An approach of in vitro testing sunflower for resistance to Sclerotinia sclerotiorum (Lib.) de Bary. Romanian Agricultural Research, 2: 35-39.

Roberts, N., Vear, F. and Tourvieille de Labrouche, D., 1987. L'heredite de la resistance au Sclerotinia sclerotiorum (Lib.) de Bary chez le tournesol. Etude des reactions à deux test mycelins. Agronomie, pp. 423-429.

Škorić, D. and Rajčan, I., 1992. Breeding for Sclerotinia resistance in sunflower. Proceedings of the $13^{\text {th }}$ International Sunflower Conference, Pisa, Italy, pp. 1257-1262.

$\mathrm{Tu}$, J.C., 1985. Tolerance of white bean (Phaseolus vulgaris) to white mold (Sclerotinia sclerotiorum) associated with tolerance to oxalic acid. Physiological Plant Pathology, 26: 111-117.

Vasić, D., Vasiljević, Lj., Škorić, D., Malidža, G. and Glušac, D., 1997. Estimation of in vitro method of screening of effect of herbicides on sunflower. Proc $48^{\text {th }}$ Internat. Symposium Crop Protection, Gent., Belgium, p. 64.

Vasić, D., Alibert, G. and Škorić, D., 1999. In vitro screening of sunflower for resistance to Sclerotinia sclerotiorum (Lib.) de Bary. Helia, 22(31): 95-104.

\section{UTILIZACION DEL ACIDO OXALICO PARA LA INVESTIGACION IN VITRO DE LA RESISTENCIA A Sclerotinia EN LAS PLANTAS DE GIRASOL NO DANADAS}

\author{
RESUMEN
}

Tres lineas consaguineas del girasol que son reciprocamente diferentes en el grado de resistencia a Sclerotinia eran cultivadas en el fondo nutritivo al cual eran anadidas diversas cantidades de acido oxalico. Depues de dos semanas del cultivo, eran medidas la alteza de la planta, masa de la parte fresca y seca de superficie, el largo de la raiz y la masa de la raiz fresca y seca. Los datos obtenidos eran analizados por medio de los testes ANOVA y LSD. Entre los parametros investigados, la alteza de la planta y el largo de la raiz eran los indicadores mas ciertos de la resistencia/sensibilidad a Sclerotinia. A base de los datos obtenidos, las concetraciones de acido oxalico de $4 \mathrm{mM}, 3 \mathrm{mM}$ y $2 \mathrm{mM}$ fueron seleccionadas para el trabajo ulterior durante el cual sera investigado el numero considerable de los genotipos del girasol. 
UTILISATION DE L'ACIDE OXALIQUE DANS L'EXAMEN IN VITRO DE LA RÉSISTANCE ENVERS LA Sclerotinia SUR DES PLANTES DE TOURNESOL INTACTES

\author{
RÉSUMÉ
}

Trois lignes inbred différentes par leur résistance à la Sclerotinia ont été cultivées dans un milieu nutritif auquel ont été ajoutées différentes concentrations d'acide oxalique. Après deux semaines de culture, la hauteur de la plante, la masse des parties aériennes fraîche et sèche, la longueur de la première paire de feuilles, la longueur de la racine et la masse de racine fraîche et sèche ont été évaluées. Les données obtenues ont été analysées au moyen de tests ANOVA et LSD. Parmi les paramètres examinés, ce sont la hauteur de la plante et la longueur de la racine qui sont apparues comme les indicateurs les plus sûrs de la résistance ou de la sensibilité à la Sclerotinia. À partir des données obtenues, les concentrations d'acide oxalique de $4 \mathrm{mM}, 3 \mathrm{mM}$ et $2 \mathrm{mM}$ ont été choisies pour des recherches ultérieures au cours desquelles on pourra tester un plus grand nombre de génotypes. 
